\section{SAT0393 DEVELOPMENT OF A SCORE FOR THE DIAGNOSIS OF INFECTIOUS ARTHRITIS IN DIFFICULT TO PUNCTURE JOINTS}

C.A. Guillen-Astete, B. Blanco Cáceres. Rheumatology Department, Ramon y Cajal University Hospital, Madrid, Spain

Background: In previous studies, we have demonstrated the benefit of the determination of serum procalcitonin in the diagnostic discrimination between gouty and an infectious monoarthritis. On the other hand, the utility of this analyte has been suggested in the diagnostic study of monoarthritis in which the obtention of joint fluid for lab analysis is difficult or impossible in the immediate term.

Objectives: The objective of this study is to perform the validation of a score to determine the diagnosis of Native Joint Septic Arthritis (ASAN) in difficult to puncture joints.

Methods: A logistic regression study was conducted using 37 cases of ASAN (sternoclavicular, acromioclavicular, coxofemoral, intratarsal, and metatarsophalangeal) and 160 of non-infectious arthritis diagnosed as such between 2013 and 2016 by microbiological criteria, the presence of crystals or absence of both situations. The explanatory variables were: Three age strata, three CRP levels, three strata of PCT figure, immunomodulation/immunosuppression condition, two strata of time of evolution, the presence of fever and neutrophilia. After a forward modelling, a test validation study was performed using the modelling coefficients as a weight reference for each value of the score.

Results: The only variables that overcame the forward modelling were PCT, temperature, immunosuppression and time of evolution. Using the reference coefficients (table 1), validation tests were performed by means of the ROC curve. According to the presented curve, a sensitivity of $86.6 \%$ and specificity of $95.8 \%$ is reached if the total score reaches or exceeds 5 pts (Strata of PCT 6, 4 and 2 , and 2 points by any of the remaining three considerations). The total area under the curve was 0.926 .

Abstract SAT0393 - Table 1. Results of the multivariable logistic regression backward modelling.

\begin{tabular}{|l|l|l|l|l|l|}
\hline Variable & Coef. & E.E. & Wald & p-value & R \\
\hline PCT $>1.4 \mathrm{ng} / \mathrm{mL}$ & 5.8412 & 1.0231 & 32.5971 & $<0.0001$ & 0.4010 \\
\hline PCT $<1.0-1.4 \mathrm{ng} / \mathrm{mL}>$ & 5.2632 & 1.9117 & 7.5798 & 0.0059 & 0.1712 \\
\hline PCT $<0.5-1.0 \mathrm{ng} / \mathrm{mL}>$ & 4.6626 & 1.7378 & 7.1985 & 0.0073 & 0.1653 \\
\hline Body temp $>38.0^{\circ} \mathrm{C}$ & 2.1119 & 0.8883 & 5.6524 & 0.0174 & 0.1385 \\
\hline Immunomodulation & 1.9623 & 0.9473 & 4.2911 & 0.0383 & 0.1097 \\
\hline Time of onset $<72 \mathrm{~h}$ & 1.6565 & 0.7649 & 4.6897 & 0.0303 & 0.1189 \\
\hline
\end{tabular}

Conclusions: Taking into account the high specificity achieved, we the authors propose the use of the present score to exclude ASAN in situations in which access to synovial fluid is difficult or technically not feasible.

Disclosure of Interest: None declared

DOI: 10.1136/annrheumdis-2018-eular.7292

\section{SAT0394 IMMUNOGENICITY AND SAFETY OF 23-VALENT PNEUMOCOCCAL VACCINE IN RA PATIENTS: RESULTS OF A 4- YEAR FOLLOW UP STUDY}

D. Bukhanova, M. Sergeeva, B. Belov, G. Tarasova, M. Cherkasova, Y. Muraviev, G. Lukina, N. Demidova. V.A. Nasonova Research Institute of Rheumatology, Moscow, Russian Federation

Background: Comorbid infections have significant impact on morbidity and mortality, especially in autoimmune diseases. Prevention of infection is an integral part of supervision of these patients.

Objectives: To investigate immunogenicity and safety of 23-valent polysaccharide pneumococcal vaccine in patients with rheumatoid arthritis (RA) treated with diseases modifying anti rheumatic drugs (DMARDs) and biologic diseases modifying anti rheumatic drugs (bDMARDs).

Methods: The study included 110 patients (females-81 (73,6\%), males-29 $(26,4 \%)$, aged $23-76 \mathrm{y}), 79 \mathrm{RA}$ pts and 31 controls with $\geq 2$ recent episodes of upper respiratory tract infections (bronchitis, pneumonia). 52 RA pts were treated with methotrexate (MT), 14 - with leflunomide (Lef), 13- with TNF- $\alpha$ inhibitors + MT. One dose $(0,5 \mathrm{ml})$ of 23-valent polysaccharide pneumococcal vaccine was administered subcutaneously without discontinuing MT/Lef or 28-30 days prior to initiation of TNF- $\alpha$ inhibitors. Control visits were scheduled as follows: at baseline (Visit I), and in 1, 3, and 12 months after immunisation. 39 out of 110 pts were followed for 24 months, 23 pts - for 36 months, and 16 pts - for 48 months. Standard clinical examination and lab tests were performed at each visit. Levels of serum antibodies $(A B)$ to Pneumococcal capsular polysaccharide were measured with VaccZymeTM PCP IgG 2 panels (The Binding Site Group Ltd, Birmingham, UK). Coefficient of post-immunisation response (CPR) was determined for each patient as the ratio of $\mathrm{AB}$ levels at Visits II, III, IV, V, VI and VII to AB level at Visit I.
Results: There were no documented clinical or radiological symptoms of bacterial pneumonia in a single patient during the FUP. CPR dynamics in RA pts on different therapeutic regimens and in the controls is shown in the table 1.

Abstract SAT0394 - Table 1. CPR dynamics in RA pts and the controls during 1-year FUP, $M \pm \sigma$.

\begin{tabular}{lccccc}
\hline & $\begin{array}{c}\text { Visit II } \\
(1 \text { month })\end{array}$ & $\begin{array}{c}\text { Visit IV } \\
(12 \text { month })\end{array}$ & $\begin{array}{c}\text { Visit V } \\
(24 \text { month })\end{array}$ & $\begin{array}{c}\text { Visit VI } \\
(36 \text { month })\end{array}$ & $\begin{array}{c}\text { Visit VII } \\
(48 \text { month })\end{array}$ \\
\hline RA & $2,33^{*}$ & $2,64^{*}$ & $3,07^{*}$ & 1029 & $2,08^{*}$ \\
patients & {$[1,6 ; 3,8]$} & {$[2,03 ; 6,39]$} & {$[1,56 ; 6,18]$} & {$[0,74 ; 1,32]$} & {$[0,69 ; 3,80]$} \\
\hline
\end{tabular}

${ }^{*} \mathrm{p}<0,05$

Pronounced positive immune reaction to the study vaccine was documented in all RA patients on different therapeutic regimens as significant CPR incremental growth. The proportion of responders to the vaccine reached $61 \%$ among RA pts. Significant decrease of RA activity according to DAS28 scores in RA pts (4,32 and 3,31 at Visits I and IV, respectively, $\mathrm{p}<0,001$ ) demonstrates absence of any negative effect of vaccination on disease activity. There was a trend to weakening of post-vaccination response in 3 years, although significantly high level of postimmunisation response still persisted after 4 years of FUP. Not a single case of pneumococcal infection was ever documented during 4 years FUP, although one case of interstitial viral pneumonia was registered in 6 month after immunisation. Not a single vaccination-related RA exacerbation episode was documented during the FUP.

Conclusions: Therefore obtained results are indicative of sufficient immunogenicity, good safety and efficacy of 23 -valent pneumococcal vaccine in RA pts on different therapeutic regimens.

Disclosure of Interest: None declared

DOI: 10.1136/annrheumdis-2018-eular.1586

\section{SAT0395 ASSESSMENT OF EFFICACY AND SAFETY OF A TRIVALENT SPLIT-VIRUS INFLUENZA VACCINE IN PATIENTS WITH RHEUMATIC DISEASES}

D. Bukhanova, B. Belov, G. Trasova, S. Erdes, T. Dubinina, G. Lukina, N. Demidova, A. Volkov, N. Yudkina. V.A. Nasonova Research Institute of Rheumatology, Moscow, Russian Federation

Background: In current rheumatology practice concurrent infections produce significant negative impact on patients' morbidity, mortality and quality of life, especially in cases of systemic connective tissue diseases. Based on WHO estimations the annual incidence of influenza in adult population amounts to $5 \%-10 \%$ worldwide. Influenza can lead to hospitalisation ( 3 to 5 million cases per year) and even death (250-500 thousand cases per year). Flu and its complications rates are higher in patients with rheumatic diseases (RD) as compared to general population. Therefore, prevention of influenza should be viewed as integral part of RD population management.

Objectives: To study the safety and efficacy of inactivated split-virus influenza vaccine in patients with rheumatoid arthritis (RA), ankylosing spondylitis (AS), and systemic scleroderma (SS) treated with diseases modifying anti rheumatic drugs (DMARDs) and biologic diseases modifying anti rheumatic drugs (bDMARDs).

Methods: 133 subjects ( 97 females and 36 males, aged 22-82 y) with recent acute respiratory viral infections $(\mathrm{ARVI})$ and flu episodes in medical records were enrolled, including 52 RA patients, 34 AS patients, 7 SS patients and 40 healthy volunteers as the control group.

39 RA pts received methotrexate (MTX), 12 - TNF $\alpha$ inhibitors+MTX, 8 - leflunomide, 2 - abatacept, 2 - sulfasalazine, 1 - tofacitinib +MTX. 19 AS patients were treated with nonsteroidal anti-inflammatory drugs (NSAIDs), 15 -with TNF $\alpha$ inhibitors. The RD duration ranged from 2 months to 46 years. All participants were injected subcutaneously with one dose $(0.5 \mathrm{ml})$ of the "Vaxigrip" vaccine containing the actual influenza virus strains with ongoing therapy. The control visits were scheduled at baseline, and in 1, 3 and 6 months after vaccination (Visits 0, 1, 2 and 3 , respectively). Standard clinical and laboratory tests were performed during each visit.

Results: Vaccine tolerability was good in 103 participants (77,4\%). Post-vaccination pain, swelling and redness of the skin up to $2 \mathrm{~cm}$ in diameter were registered in 20 cases (15\%), low-grade fever, myalgia and malaise were documented in 10 cases $(7,5 \%)$. There was no causal relationship between these reactions and principal therapy, therefore, no modifications of therapeutic regimens were required, and complete resolution occurred within 24 hours without additional interventions. No RD exacerbations or emergence of de novo autoimmune disorders were observed during the FUP. At baseline mean pts' DAS28 and BASDAI scores were 3.56 and 3.85 , improving up to 1.99 and 3,09, respectively, 6 mo post-vaccination. For the entire FUP there were no cases of influenza or influenza-like illness registered. 